\title{
Study on Piezoelectric Actuators in Vibration Control of a Planar Parallel Manipulator
}

\author{
Bongsoo Kang ${ }^{1}$ and James K. Mills ${ }^{2}$ \\ ${ }^{1}$ Department of Mechanical Engineering, Hannam University \\ Daejeon, Korea 306-791, bskang@hannam.ac.kr \\ ${ }^{2}$ Department of Mechanical and Industrial Engineering, University of Toronto \\ Toronto, Ontario, Canada M5S 3G8, mills@ mie.utoronto.ca,
}

\begin{abstract}
This paper presents a new approach for the use of piezoelectric materials, PVDF and PZT, for vibration attenuation of a planar parallel manipulator. Since lightweight linkages of parallel manipulators deform under high acceleration/deceleration, such motion induced vibration would have to be damped quickly to reduce settling time of the platform position. An active damping controller is developed, which consists of a PD feedback control scheme, applied to linear actuators for rigid body motions, and a linear velocity feedback (L-type) scheme applied to either PVDF layer or PZT actuators for vibration attenuation. Simulation results show that piezoelectric materials yield good damping performance, resulting in precise manipulations of the planar parallel manipulator.
\end{abstract}

\section{Introduction}

Interest in parallel manipulators has been grown recently. Since the platform of parallel manipulators is supported by multiple links, its structure has high mechanical rigidity and low inertia of moving components, resulting in high positioning accuracy and fast movements [1][2]. In electronic industry, where small product size and short assembly time characterize the manufacturing process, a high speed pick-and-place position mechanism is an indispensable element. Therefore, the parallel mechanism provides an alternative design to conventional serial mechanisms, such as gantry robots, for the application of integrated circuit production.

The major problems of a parallel manipulator are small workspace and associated singularities. Hence, considerable researches have focused on kinematic analysis and singularity characterization of these devices [3][4]. Since parallel mechanisms generate multiple closed-chains between linkages, its dynamic modeling is difficult to achieve, especially when the Newton-Euler method is applied [5]. Applying the Lagrangian method or the principle of virtual work to parallel mechanisms, makes it easy to analyze dynamic behaviors of parallel mechanisms [6]. Moreover, the electronics fabrication industry demands high-speed, high acceleration placement machines, with corresponding lightweight linkages, hence, these linkages deform under high inertia forces. As a result, modeling structurally flexible linkages, which make up closed-loop kinematic chains of parallel manipulators, has been the focus of substantial attention in the literature [7][8].

In this paper, an active damping approach using piezoelectric materials is presented to damp out vibration of a planar parallel manipulator. Attached on the surface of flexible linkages, piezoelectric materials deform under a control voltage applied, and produce shear force, which can counteract shear stress occurred due to deformation of linkages. Transducers are often developed from either of two types of material: polyvinylidene fluoride (PVDF) and lead zirconium titanate (PZT). PVDF is much more lightweight, but PZT can yield better performance in vibration damping since PZT has higher strain constant than PVDF. PVDF are configured as a uniform layer, with control voltage applied to the overall layer. Several small PZT segments can be attached on the linkage together, and controlled separately. The control system of a planar parallel manipulators consists of two parts. The first part is a PD feedback control scheme to make the platform track a given trajectory. The second part is a linear velocity feedback (L-type) scheme [9] to control voltages applied to piezoelectric materials. Simulation results show that piezoelectric materials attenuate vibration of linkages effectively, so that settling time of the platform position is reduced remarkably.

\section{Architecture of Planar Parallel Manipulator}

The architecture of the considered planar parallel manipulator is illustrated in Figure1. The moving platform, with revolute joins on each vertex, exhibits translation and rotational motion. Three intermediate linkages, between the moving platform and sliders, play a role to convert actuating forces into movements of the platform. Both ends of three intermediate linkages are composed of non-actuated revolute joints. The motion of the sliders can 
be achieved with a ball-screw mechanism driven by a rotary motor.

The planar parallel manipulator is categorized as a $\underline{P R R}$ type, because a closed chain consists of a prismatic joint and two consecutive revolute joints, beginning from the prismatic joint of the slider to the revolute joint between the linkage and the platform. In contrast to the wellknown Stewart-Gough platform[2], the driving actuator are stationary, as shown in Figure 1, hence are not included in the total inertia of moving components.

\section{Dynamic Model of Flexible Linkage}

Generalized coordinates for the planar parallel manipulator are assigned as shown in Figure 2. The position and orientation, (pose) of the platform at its center, $P$, with respect to a reference frame $\mathrm{X}-\mathrm{Y}$, shown in Figure 2, is defined as

$$
\mathbf{X}_{P} \equiv\left[x_{P}, \quad y_{P}, \quad \phi\right]^{T} \in \mathfrak{R}^{3}
$$

The origin of the reference frame is placed at the point where imaginary extended lines of three actuators intersect, as shown in Figure 2. The placement of the sliders from their origin, $A_{i}$, to $B_{i}$, is expressed as

$$
\boldsymbol{\rho} \equiv\left[\begin{array}{lll}
\rho_{1}, & \rho_{2}, & \rho_{3}
\end{array}\right]^{T} \in \mathfrak{R}^{3}
$$

Finding positions of the sliders corresponds to an inverse kinematic solution when the pose of the platform is given. $\beta_{i}$ is defined as the angle between the $\mathrm{X}$-axis of the fixed frame and the $i^{\text {th }}$ intermediate link at $B_{i}$ and $\alpha_{i}$ is the constant angle between the $\mathrm{X}$-axis and the $i^{\text {th }}$ linear actuator. Each vertex of the platform is denoted as $C_{i}$. Three linkages, including associated coordinates, are numbered with a subscript, $i$, starting from the lower right linkage.

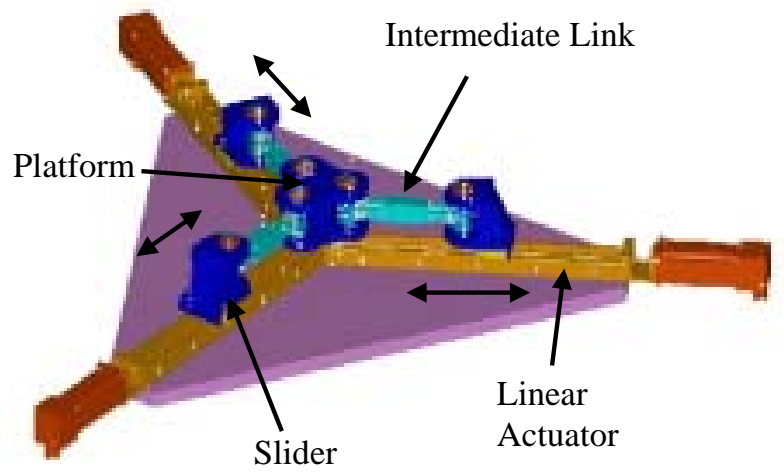

Figure 1: Configuration of the manipulator with motion of sliders indicated

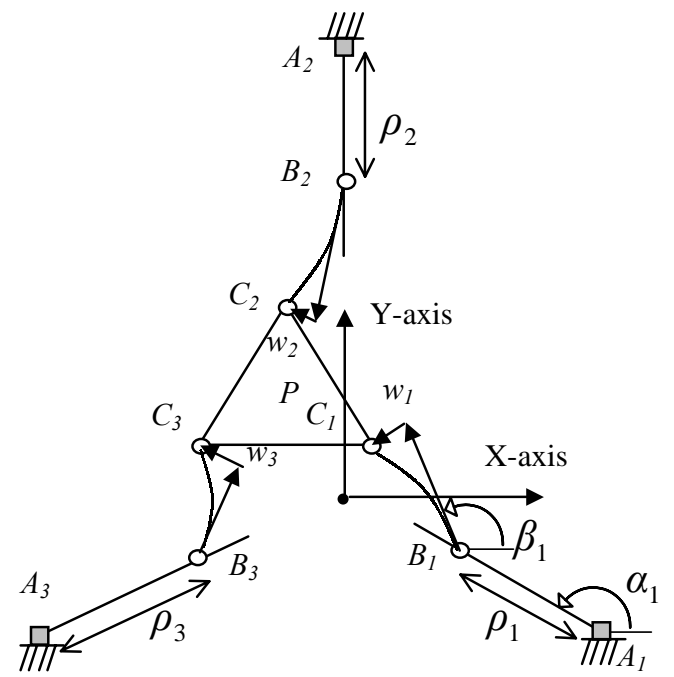

Figure 2: Schematic illustrating the coordinates of the parallel manipulator. Note the linkages $B_{i} C_{i}, i=1,2,3$ are shown with exaggerated deformations.

The three linkages are assumed to exhibit structural flexibility, hence the shape of linkages, under high acceleration, are shown deformed, in Figure 2. The lateral deformation of $i^{\text {th }}$ linkages from its undeformed distal end, $C_{i}$, is denoted as $w_{i}$. Out of $\mathrm{X}-\mathrm{Y}$ plane deformations are not considered in this paper. Utilizing an assumed modes model[10], these lateral flexible deformations are expressed as follows;

$$
w_{i}(x, t)=\sum_{j=1}^{r} \eta(t)_{i j} \psi_{j}(\xi) i=1,2,3
$$

where: $\xi$ is non-dimensional coordinates along the link. The subscript, $j$, implies the $j^{\text {th }}$ vibration mode and $r$ represents a finite number of assumed modes. Assuming that both ends of the link exhibit pinned-free motion at $B_{i}$ and $C_{i}$ respectively, normalized shape functions, satisfying these boundary condition, are selected as:

$\psi_{j}(\xi)=\frac{1}{2 \sin \left(\gamma_{j}\right)}\left[\sin \left(\gamma_{j} \xi\right)+\frac{\sin \left(\gamma_{j}\right)}{\sinh \left(\gamma_{j}\right)} \sinh \left(\gamma_{j} \xi\right)\right]$

where: $\gamma_{j}=(j+0.25) \pi \quad j=1,2, \ldots, r$

Figure 3 shows the first three vibration modes and their derivatives with respect to $x$.

The generalized coordinates of the platform are denoted, in vector form as:

$$
\boldsymbol{X} \equiv\left[\begin{array}{llll}
\boldsymbol{\rho}, & \boldsymbol{\beta}, & \boldsymbol{X}_{\boldsymbol{P}}, & \boldsymbol{\eta}
\end{array}\right]^{T} \in \mathfrak{R}^{9+3 r}
$$

where: $\boldsymbol{\beta} \equiv\left[\begin{array}{lll}\beta_{1}, & \beta_{2}, & \beta_{3}\end{array}\right]^{T} \in \mathfrak{R}^{3}$ 
$\boldsymbol{\eta} \equiv\left[\begin{array}{lllllll}\eta_{11} & \cdots & \eta_{21} & \cdots & \eta_{31} & \cdots & \eta_{3 r}\end{array}\right]^{T} \in \mathfrak{R}^{3 r}$

Using Lagrangian equation of the first type, with generalized coordinates defined previously, the equation of motion for the planar parallel manipulator are complete with a total of $9+3 r$ equation, [8] given below;

$$
\begin{gathered}
{\left[\begin{array}{cccc}
M_{11} & M_{12} & 0 & M_{14} \\
M_{12}^{T} & M_{22} & 0 & M_{24} \\
0 & 0 & M_{33} & 0 \\
M_{14}^{T} & M_{24}^{T} & 0 & M_{44}
\end{array}\right]\left[\begin{array}{c}
\ddot{\rho} \\
\ddot{\beta} \\
\ddot{X}_{P} \\
\ddot{\eta}
\end{array}\right]+\left[\begin{array}{c}
V_{1} \\
V_{2} \\
0 \\
V_{4}
\end{array}\right]+} \\
{\left[\begin{array}{llll}
0 & 0 & 0 & 0 \\
0 & 0 & 0 & 0 \\
0 & 0 & 0 & 0 \\
0 & 0 & 0 & K
\end{array}\right]\left[\begin{array}{c}
\rho \\
\beta \\
X_{P} \\
\eta
\end{array}\right]=\left[\begin{array}{l}
U \\
\ldots \\
0
\end{array}\right]+\left[\begin{array}{l}
J_{\Gamma 1} \\
J_{\Gamma 2} \\
J_{\Gamma 3} \\
J_{\Gamma 4}
\end{array}\right]}
\end{gathered}
$$

where: $\boldsymbol{M}_{11} \in \mathfrak{R}^{3 \times 3}, \boldsymbol{M}_{12} \in \mathfrak{R}^{3 \times 3}, \boldsymbol{M}_{14} \in \mathfrak{R}^{3 \times 3 r}, \boldsymbol{M}_{22} \in \mathfrak{R}^{3 \times 3}$,

$$
\begin{aligned}
\boldsymbol{M}_{24} \in \mathfrak{R}^{3 \times 3 r}, \boldsymbol{M}_{33} \in \mathfrak{R}^{3 \times 3}, \boldsymbol{M}_{44} \in \mathfrak{R}^{3 r \times 3 r} \\
\quad \equiv \text { inertia matrices } \\
\boldsymbol{V}_{l} \in \mathfrak{R}^{3}, \boldsymbol{V}_{2} \in \mathfrak{R}^{3}, \boldsymbol{V}_{4} \in \mathfrak{R}^{3 r} \\
\quad \equiv \text { vectors of Coriolis and centrifugal terms } \\
\boldsymbol{K} \in \mathfrak{R}^{3 r \times 3 r} \equiv \text { stiffness matrix of the linkages } \\
\boldsymbol{\lambda} \in \mathfrak{R}^{6} \equiv \text { vector of Lagrange multipliers } \\
\boldsymbol{J}_{\Gamma 1} \in \mathfrak{R}^{3 \times 6}, \boldsymbol{J}_{\Gamma 2} \in \mathfrak{R}^{3 \times 6}, \boldsymbol{J}_{\Gamma 3} \in \mathfrak{R}^{3 \times 6}, \boldsymbol{J}_{\Gamma 4} \in \mathfrak{R}^{3 r \times 6} \\
\quad \equiv \text { partial derivatives of constraint equations with } \\
\text { respect to the corresponding generalized coordinate } \\
\boldsymbol{U} \equiv\left[\begin{array}{lll}
u_{1} & u_{2} \quad u_{3}
\end{array}\right]^{T} \in \mathfrak{R}^{3} \\
\equiv \\
\equiv \text { vector of control inputs for linear actuators }
\end{aligned}
$$

Details of each components in the above are given in [8].
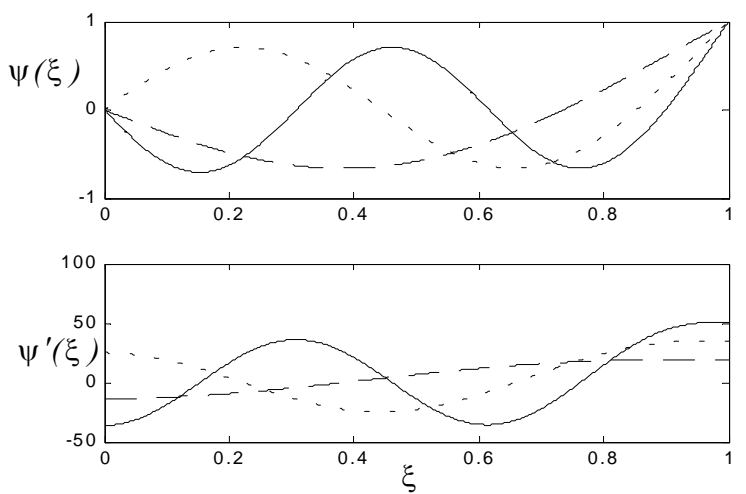

Figure 3: First three mode shapes of straight beam and its derivatives with respect to $x$

(dashed line: first, dotted line: second, solid line: third)

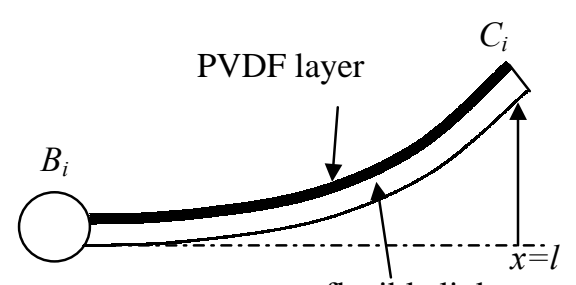

flexible link

Figure 4(a): Intermediate link with PVDF layer

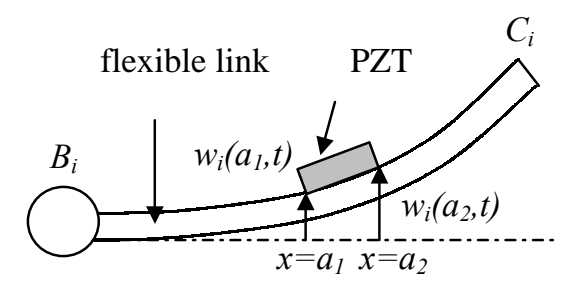

Figure 4(b): Intermediate link with PZT actuator

\section{Control Design}

If the intermediate link is very stiff, an appropriate rigid body control method for three linear actuators can yield good tracking performance of the platform. However, structural flexibility of the linkages transfers unwanted vibration to the platform, and may even lead to instability of the system. Since it's hard for three linear actuators to achieve trajectory tracking of the platform and vibration attenuation of linkages simultaneously, an active damping approach is proposed through the use of smart material, a PVDF layer and a PZT segment. Attached on the surface of the linkage, piezoelectric materials generate shear force under control voltage applied, which can oppose shear stress occurred due to elastic deformation of the linkages.

A simple proportional and derivative (PD) feedback controller is used for three linear actuators and is given as

$$
u_{i}(t)=-k_{P}\left(\rho_{d i}-\rho_{i}\right)-k_{d}\left(\dot{\rho}_{d i}-\dot{\rho}_{i}\right), \quad i=1,2,3
$$

where $k_{p}$ and $k_{d}$ are a proportional and a derivative feedback gain respectively. $\rho_{d i}$ and $\dot{\rho}_{d i}$ are desired displacement and velocity of the $i^{\text {th }}$ slider respectively. Following sections give an active damper using piezoelectric materials.

\subsection{PVDF}

PVDF layers can be bonded uniformly on the one side of the linkages of the planar parallel manipulator, as shown in Figure 4(a). When a control voltage, $V_{i}$, is applied, the virtual work done by the PVDF layer is expressed as 


$$
\delta W_{P V D F}=c V_{i}(t) \sum_{j=1}^{r} \psi_{j}^{\prime}(l) \delta \eta_{i j}
$$

where $c$ is a constant representing the bending moment per volt and $\left(y^{\prime}\right.$ implies differentiation with respect to $x$. If the control voltage, $V_{i}$, is selected as

$$
V_{i}(t)=-k_{I} \dot{w}_{i}^{\prime}(l, t) \quad i=1,2,3
$$

the slope velocity, $\dot{w}^{\prime}(l, t)$, converges to zero, hence vibration of linkages damped out. Since the slope velocity, $\dot{w}^{\prime}(l, t)$, is not easily available, an alternative scheme, the

L-type method [9], is given as

$$
V_{i}(t)=-k_{I} \dot{w}_{i}(l, t) \quad i=1,2,3
$$

Instead of the slope velocity, $\dot{w}^{\prime}(l, t)$, the linear velocity, $\dot{w}(l, t)$, is employed in formulating the control law. The linear velocity, $\dot{w}(l, t)$, can be achieved through the integration of the linear acceleration measured by an accelerometer installed at the distal end of the linkages, $C_{i}$. Since, at the distal end of the linkages, $C_{i}$, the shape function, $\psi_{j}(\xi=1)$, and its derivative, $\psi_{j}^{\prime}(\xi=1)$ have same trend of variation in all vibration modes, as shown in Figure 3 ;

$$
\psi_{j}(\xi=1) \psi_{j}^{\prime}(\xi=1) \geq 0 \quad j=1,2,---, r
$$

the control system keep stability even when employing the L-type method to formulate the control voltages, $V_{i}$ [9].

\subsection{PZT}

PZT is manufactured with relatively small size, hence several PZT segments can be bonded on a flexible linkage to enhance damping effect. Assuming that only one PZT segment is attached on the intermediate linkages of the manipulator, as shown in Figure 4(b), the virtual work done by the PZT actuator is expressed as

$$
\delta W_{P Z T}=c V_{i}(t) \sum_{j=1}^{r}\left[\psi_{j}^{\prime}\left(a_{2}\right)-\psi_{f}^{\prime}\left(a_{1}\right)\right] \delta \eta_{i j}
$$

$a_{1}$ and $a_{2}$ denote positions of both ends of the PZT actuator measured from $B_{i}$ along the linkage, as shown in Figure 4(b). As the PVDF layer is, the PZT actuator is controlled using the L-type method as

$$
V_{i}(t)=-k_{I}\left[\dot{w}_{i}\left(a_{2}, t\right)-\dot{w}_{i}\left(a_{1}, t\right)\right] \quad i=1,2,3
$$

In contrast to the PVDF layer, the performance of the L-type scheme for the PZT actuator depends on the location of the PZT actuator. In order to achieve stable control performance, the PZT actuator should be placed in a region on the linkage where $\psi_{j}(x)$ and $\psi_{j}^{\prime}(x)$ have the same trend of variation within $x \in\left[a_{1}, a_{2}\right]$ [9];

$$
\left(\psi_{j}\left(a_{2}\right)-\psi_{j}\left(a_{1}\right)\right)\left(\psi_{j}^{\prime}\left(a_{2}\right)-\psi_{j}^{\prime}\left(a_{1}\right)\right) \geq 0
$$

Application of this condition to vibration modes of higher frequency is restricted because satisfaction of equation (14) is only achieved in small regions on the linkage, for high frequencies.

\section{Simulation Results}

Simulations are performed to investigate damping performance of piezoelectric materials in the planar parallel manipulator with structurally-flexible linkages. Specifications of the manipulator are listed in Table 1 . The first three modes are considered in the dynamic model, i.e. $r=3$. A sinusoidal function with smooth acceleration and deceleration is chosen as the desired trajectory;

$$
x_{P}=\frac{x_{f}}{t_{f}} t-\frac{x_{f}}{2 \pi} \sin \left(\frac{2 \pi}{t_{f}} t\right)
$$

Considering the target-performance in an electrical assembly process, such as wire bonding in integrated circuit fabrication, the goal for the platform is to move linearly $2 \mathrm{~mm}\left(x_{f}\right)$ within $10 \mathrm{msec}\left(t_{f}\right)$. The trajectory is defined in the direction of the $\mathrm{X}$-axis and feedback gains are listed in Table 2 . The feedback gain, $k_{I}$, is properly selected so that the control voltage, applied to the PVDF layer, does not take over 600 Volts.

A fourth order Runge-Kutta method was used to integrate the ordinary differential equations at the integration interval of $1 \mathrm{msec}$, using MATLAB ${ }^{\mathrm{TM}}$ software. Parameters of piezoelectric materials are listed in Table 3. The placement position of the PZT actuator is adjusted as $a_{1}=0.66, a_{2}=0.91$, so that the first two vibration modes can satisfy equation (14).

Results of the PVDF layer are shown in Figures 5-8. Figure 5 shows that the error profile of the platform exhibits large oscillation at the initial acceleration, but continuously decreases due to the damping effect of the PVDF layer. The error profile of the platform without either of PVDF or PZT, labeled as "no damping" in Figure 5, shows typical characteristics of an undamped system. With Figure 6 showing deformation of the linkages on $C_{i}$, it reveals that the PVDF layer can damp structural vibration of the linkages. The first three vibration modes are illustrated in Figure 7 . The first mode has twenty times the amplitude that the second mode, and one hundred times the amplitude than the third mode. The control output for the first linear actuator is shown in the 
upper plot of Figure 8, and control voltage for the first PVDF layer is shown in the lower plot of Figure 8.

Results of the PZT actuator are shown in Figures 9-13. Comparing Figure 9 with Figure 5, the PZT actuator exhibits better damping performance than the PVDF layer. The error profile of the platform, with the PZT actuator activated, enters steady state quickly, and does not exhibit any vibration in steady state. The structural vibrations of the linkages, illustrated in Figure 10, are completely removed after $60 \mathrm{msec}$. The first three vibration modes are shown in Figure 11. The first mode has ten times the amplitudes than the other modes. Since the PZT actuator has higher strain constant than the PVDF, the PZT actuator can generate large shear force with relatively small voltage applied. The maximum voltage of the lower plot of Figure 12 is about 200 Volts, while that of the Figure 8 reaches 600 Volts. Because of dimensional restriction, only the first two modes satisfy the stability condition, given by equation (14). However, this does has little effect on damping performance, as shown in Figure 10 since the first two modes play dominant roles in vibration.

\section{Conclusions}

This paper presents a new approach for the use of piezoelectric materials, PVDF and PZT, for vibration attenuation of a planar parallel manipulator. With a L-type control scheme determining a control voltage applied, the piezoelectric materials can provide good damping performance to counteract structural vibration of flexible linkages, hence settling time of the platform of the planar parallel manipulator is reduced remarkably. Simulation results show that the planar parallel manipulator, with the lightweight linkages, undergoes persistent vibration during fast motion. Additionally, the PZT actuator, with carefully considering the segment placement, yields better performance in vibration attenuation than the PVDF layer.

\section{References}

[1] C. M. Gosselin, S. Lemieux, and J.-P. Merlet, "A new architecture of planar three-degree-of-freedom parallel manipulator," Proc. IEEE Int. Conf. on Rob. and Autom., Minneapolis, Minnesota, pp. 3738-3743, 1996.

[2] D. Stewart, "A platform with six degrees of freedom," Proc. Institution of mechanical engineers, 180 (5): 371-378, 1965.

[3] C. M. Gosselin and M. Jean, "Determination of the workspace of planar parallel manipulators with joint limits," Robotics and Autonomous Systems, 17: 129138, 1996.

[4] C. Gosselin and J. Angeles, "Singularity analysis of closed-loop kinematic chains," IEEE Trans. on Robotics and Automation, 6(3): 281-290, 1990.
[5] W. Q. D. Do and D. C. H. Yang, "Inverse dynamic analysis and simulation of a platform type of robot", J. Robotic Systems, 5(3): 209-227, 1988.

[6] C. D. Zhang and S. M. Song, "An effective method for inverse dynamics of manipulators based on the virtual work principle,” J. Robotic Systems, 10 (5): 605-627, 1993.

[7] A. Fattah, et al., "Dynamics of a 3-DOF spatial parallel manipulator with flexible links," Proc. IEEE Int. Conf. on Rob. and Autom., Nagoya Japan, pp. 627-632, 1995

[8] B. Kang and J. K. Mills, "Dynamic modeling of structurally-flexible planar parallel manipulator," Robotica, 20: 329-339, 2002.

[9] D. Sun and J. K. Mills, "PZT actuator placement for structural vibration damping of high speed manufacturing equipment," Proc. of the American Cont. Conf., San Diego, pp. 1107-1111, 1999.

[10] G. Genta, Vibration of structures and machines, Springer-Verlag New York, Inc.,1993

\begin{tabular}{ccc}
\hline Platform & $\begin{array}{c}\text { side length } \\
\text { mass }\end{array}$ & $\begin{array}{c}100 \mathrm{~mm} \\
0.2 \mathrm{~kg}\end{array}$ \\
\hline Slider & mass & $0.2 \mathrm{~kg}$ \\
\hline Linear & stroke & $400 \mathrm{~mm}$ \\
Actuator & incline angle & $120^{\circ}, 270^{\circ}, 30^{\circ}$ \\
\hline Intermediate & length & $200 \mathrm{~mm}$ \\
Link & density & $2770 \mathrm{~kg} / \mathrm{m}^{3}$ \\
& modulus & $73 \mathrm{GPa}$ \\
& cross-section & $25 \mathrm{~mm}(\mathrm{~W}) * 1.5 \mathrm{~mm}(\mathrm{H})$ \\
\hline
\end{tabular}

Table 1: Specification of the planar parallel manipulator

\begin{tabular}{cc}
\hline$k_{p}$ & $10,000 \mathrm{~N} / \mathrm{m}$ \\
$k_{d}$ & $500 \mathrm{~N}-\mathrm{sec} / \mathrm{m}$ \\
$k_{I}$ & $4,000 \mathrm{~V}$-sec/m for PVDF \\
& $1,500 \mathrm{~V}$-sec/m for PZT \\
\hline
\end{tabular}

Table 2: Feedback control gains

\begin{tabular}{ccc}
\hline & PVDF & PZT \\
\hline modulus & $2 \mathrm{GPa}$ & $63 \mathrm{GPa}$ \\
length & $200 \mathrm{~mm}$ & $50 \mathrm{~mm}$ \\
thickness & $0.28 \mathrm{~mm}$ & $0.75 \mathrm{~mm}$ \\
width & $25 \mathrm{~mm}$ & $25 \mathrm{~mm}$ \\
density & $1800 \mathrm{~kg} / \mathrm{m}^{3}$ & $7600 \mathrm{~kg} / \mathrm{m}^{3}$ \\
$\mathrm{~d}_{31}$ & $22 * 10^{-12} \mathrm{~m} / \mathrm{V}$ & $110 * 10^{-12} \mathrm{~m} / \mathrm{V}$ \\
\hline \multicolumn{3}{c}{ Table $3:$ Parameters of piezoelectric materials }
\end{tabular}




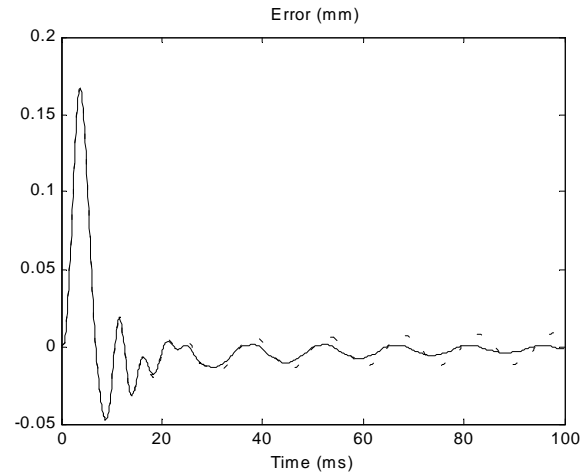

Figure 5: Error profile of the platform (dotted line: no damping, solid line: with PVDF layer)

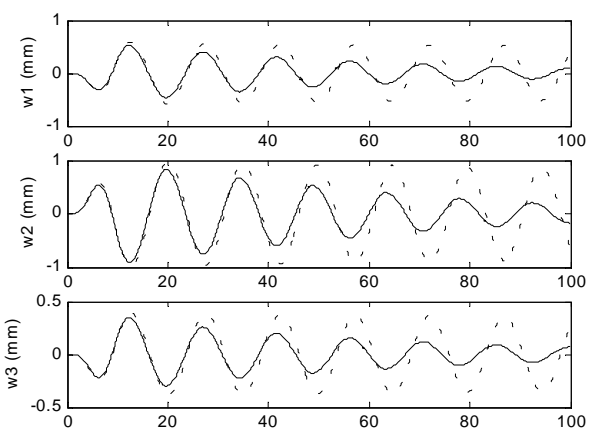

Figure 6: Flexible deformation of each intermediate link (dotted line: no damping, solid line: with PVDF layer)

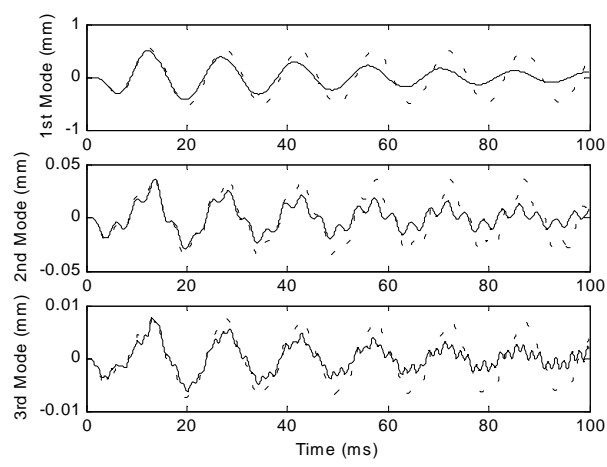

Figure 7 : The first three vibration modes of the first link (dotted line: no damping, solid line: with PVDF layer)
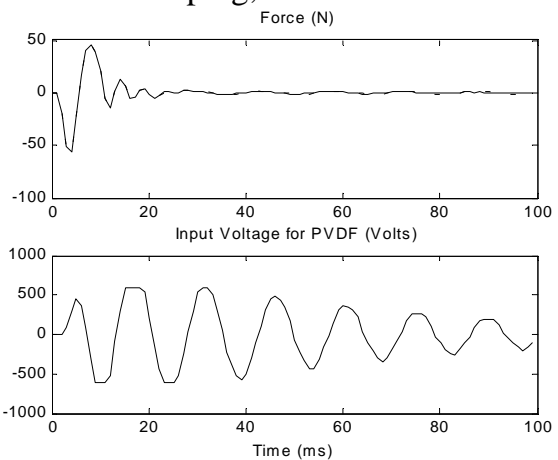

Figure 8 : Control inputs for the $1^{\text {st }}$ actuator (dotted line: no damping, solid line: with PVDF layer)

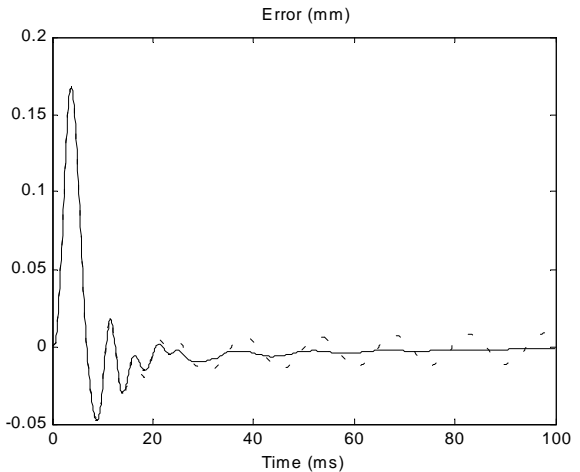

Figure 9: Error profile of platform (dotted line: no damping, solid line: with PZT actuator)

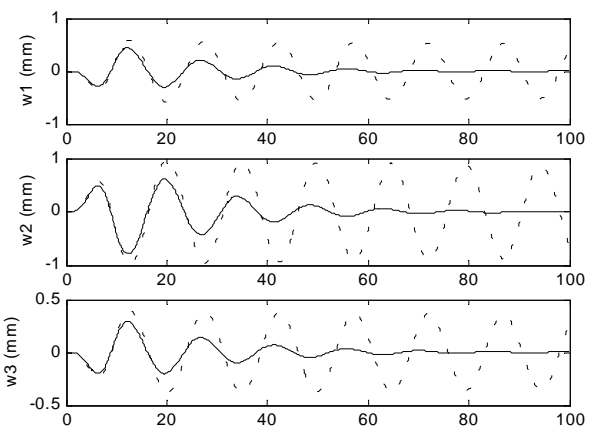

Figure 10: Flexible deformation of each intermediate link (dotted line: no damping, solid line: with PZT actuator)

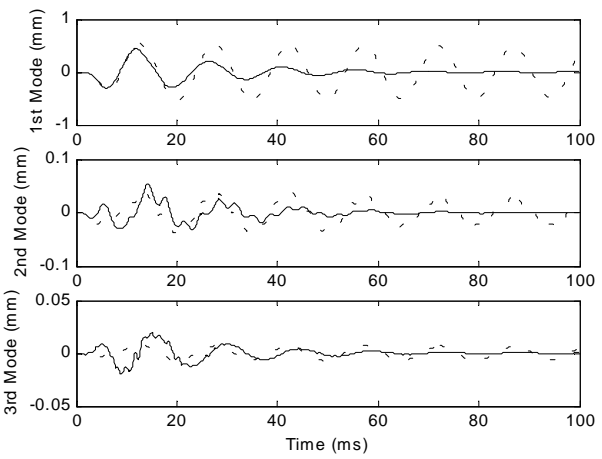

Figure 11: The first three vibration modes of the first link (dotted line: no damping, solid line: with PZT actuator)
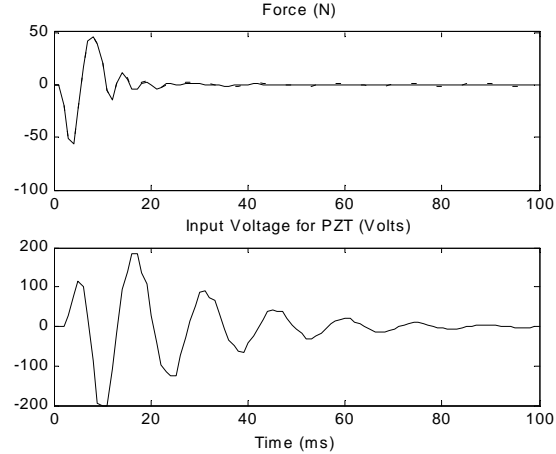

Figure 12: Control input for the $1^{\text {st }}$ intermediate link (dotted line: no damping, solid line: with PZT actuator) 